\title{
Errors of optical vitreous silica heat diffusivity determination by pulse method
}

\author{
M.D. Katz \\ Ministry of Education and Science of the Russian Federation the National Research Tomsk Polytechnic \\ University Tomsk, Russia
}

\begin{abstract}
The accuracy appraisal of thermophysical characteristics determination of optical vitreous silica was carried out by a pulse method by comparison of numerical solution results of problem of a non-stationary temperature sample field at collimated laser impulse of final duration impact on its surface and reference values of heat diffusivity.
\end{abstract}

\section{Introduction}

In recent decades, increased attention is drawn to methods for determining the heat diffusivity and thermal conductivity and heat capacity of materials under high enough temperature conditions. A method of the laser impulse [1-4] which essence consists in absorption of a frontal ("hot") surface of a radiant energy impulse sample in a thin layer and its return ("cold") surface temperature measurement registration in time represents the greatest interest for researchers.

The maximum temperature of an overheat $\left(T_{\max }\right)$, attainment time $\left(\tau_{05}\right)$ of a half the maximum temperature of "cold" (not heated) sample surface overheat is determined by the received temporary temperature dependence of the sample's return surface and heat diffusivity of a material $(a)$ is calculated using a formula [1]:

$$
a=0,1388 \cdot L^{2} / \tau_{05} .
$$

If we know the heat diffusivity, it is rather simple to determine the heat conductivity and specific heat of materials by formulas [1].

The use of this method for determination of heat diffusivity of the optical vitreous silica which is characterizing from used modifications of glasses by the limit characteristics: high temperature of a softening and evaporation, low coefficient of thermal expansion, high optical transparency and chemical firmness [5] is of known interest.

General analysis theory of heat transfer processes in partially transparent materials was developed for a long time [6-8]. The applicability of the laser pulse method to determine the heat diffusivity of silicate glass samples with different additives was studied in [9-11]. In [9] found that for conditions of small values of optical thickness and low heat transfer from the sample's surfaces it is possible to

\footnotetext{
${ }^{a}$ Corresponding author: katz@tpu.ru
}

This is an Open Access article distributed under the terms of the Creative Commons Attribution License 4.0, which permits unrestricted use, distribution, and reproduction in any medium, provided the original work is properly cited. 


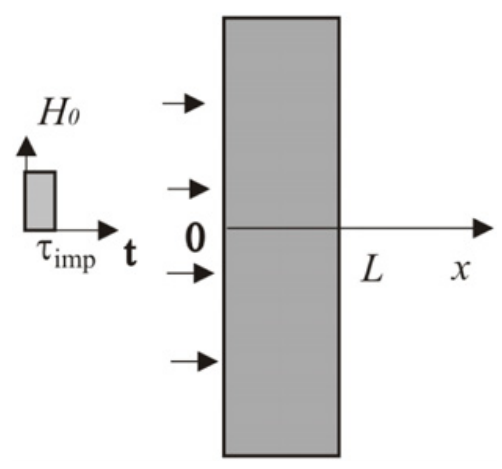

Figure 1. Scheme of solution domain.

determine the heat diffusivity of the material by laser pulse. On the basis of the developed analytical model of radiation-conductive heat transfer it was shown [10] the influence of the sample's thickness and the heat transfer from its surface on the results of silicate glass heat diffusivity measurement by the laser pulse method. Based on the analysis of heat exchange for the scattering medium, heated by diffused and collimated radiation flows it was shown [11] that the analysis of heat transfer processes can be carried out in the framework of the linear thermal conductivity models at small pulse length of the laser heating.

However, to date analysis of the quantities of methodological errors in the determination of heat diffusivity of optical vitreous silica by laser pulse was not performed. Possible intensification of heat due to radiation heat transfer can lead to the growth of errors in the determination of thermal characteristics compared with previously established ones [12] for opaque materials.

The purpose of this paper is to estimate the errors in the determination of heat diffusivity of optical vitreous silica by laser pulse method [1] due to radiation heat transfer in the sample material unrecorded at developing of method.

\section{Statement of the problem}

It was hypothesized when the problem statement that the material and the boundary surfaces of the sample are gray. The one-dimensional heat conduction problem for an infinite plate when impact of the collimated laser beam of finite time duration $\tau_{\text {imp }}$ on its surface and absence of heat exchange on the "hot" and "cold" boundaries was solved by the finite difference method.

Computational errors of heat diffusivity by laser pulse method were determined by comparing the reference values of these characteristics with the values obtained by numerical simulation of realization conditions of experiments on determination of thermal properties of materials by this method, in terms of radiation-conductive heat transfer in the sample.

Solution domain presented the endless optical vitreous silica plate with thickness $L$ (Fig. 1), surface part $x=0$ of which was heated by a collimated energy flow directed perpendicular to the plane of the plate at a density of radiation $H_{0}$ and the duration of the pulse $\tau_{\text {imp }}$.

It was assumed that the natural radiation of environment is small in comparison with the external radiation flux. Problem reduces to the solution of the energy equation (1) with boundary (2-4) and the initial (5) conditions:

$$
\rho c \frac{\partial T(x, t)}{\partial t}=\lambda \frac{\partial^{2} T(x, t)}{\partial x^{2}}-\frac{\partial H(x)}{\partial x}
$$




$$
\begin{gathered}
x=0: \quad-\lambda \frac{\partial T(x, t)}{\partial x}=H_{0}, \quad \tau_{\text {imp }}>t>0 ; \\
x=0: \quad-\lambda \frac{\partial T(x, t)}{\partial x}=0, \quad t>\tau_{\text {imp }} \\
x=L: \quad \lambda \frac{\partial T(x, t)}{\partial x}=0, \quad t>0 ; \\
t=0: \quad T(x, 0)=T_{0},
\end{gathered}
$$

where $H_{0}, H(x)$ - the radiation flux density on the heated sample's surface and the material in the coordinate direction $x ; c$ - specific heat capacity; $\lambda$ - thermal conductivity; $\rho$ - density; $\tau_{\text {imp }}-$ pulse length of laser radiation; $T, T_{0}$ - current and initial temperatures; $t$ - time.

Changing the energy density of the material was determined by the Bouguer-Lambert-Beer law [13]:

$$
H(x)=H_{0} \cdot \exp \left(-k_{\lambda} \cdot x\right),
$$

where $\mathrm{k}_{\lambda}$ - energy absorption coefficient of collimated laser radiation flow, $\mathrm{m}^{-1}$.

\section{Solution method}

The method of finite differences was used to solve the system of differential equations and boundary conditions (1)-(5). Difference analogues of differential equations and boundary conditions were solved by the sweep method using implicit iterative four-point difference scheme [14].

An iterative algorithm developed for solving the nonlinear problems of heat transfer in terms of the local intense heating $[15,16]$ was used. The verification of difference schemes conservatism by the method [17], developed for testing the heat and mass transfer problems in terms of fast phase transformations processes was conducted to justify the validity of the numerical simulation results in the absence of experimental data on the material sample's temperature fields under conditions of the heat by radiation fluxes of high intensity (appropriate to methodology [1, 2]).

\section{Results and discussion}

Simulation of experimental conditions was conducted for optical vitreous silica KU-1 with thermal characteristics: $\lambda=1,35 \mathrm{~W} /(\mathrm{m} \cdot \mathrm{K}), c=728 \mathrm{~J} /(\mathrm{kg} \cdot \mathrm{K}), \rho=2200 \mathrm{~kg} / \mathrm{m}^{3}$. Value of the initial temperature and the thickness of the sample for the considered experiments were, respectively, $T_{0}=$ $293 \mathrm{~K}$ and $L=0,003 \mathrm{~m}$.

The wavelength range of the transparency region of the vitreous silica is $2100 \ldots 2800 \mathrm{~nm}$, which corresponded to a change in the absorption coefficient from 10 to $250 \mathrm{~m}^{-1}$. Radiative heat flux values $H_{0}=5 \cdot 10^{7} \mathrm{~W} / \mathrm{m}^{2}$ and a pulse duration $\tau_{\text {imp }}=0,5 \mathrm{~ms}$ were selected according to the actual characteristics of the laser source in the experimental apparatus to determine the thermal conductivity of materials. Likewise, the basic position of the method [1] - a mandatory "temperature overheating" on "cold" sample surface at least $3 \ldots 6 \mathrm{~K}$ was taken into account at choosing these parameters. Steps of the difference grid in time and space were respectively $50 \mathrm{~ms}$ and $12 \mu \mathrm{m}$.

The dependences of error $\delta_{a}$ of vitreous silica KU-1 heat diffusivity determination on absorption coefficient $k_{\lambda}$ (Fig. 2) illustrate the influence of this effect - additional heat inflow into the deep layers of the sample due to radiative heat transfer. The values of $\delta_{a}$ are increased and they are respectively about $30 \%$ when $\mathrm{k}_{\lambda}=150 \mathrm{~m}^{-1}$ with increasing absorption coefficient.

The results of the conducted simulation provide a basis for concluding that the practical implementation of methods for determining the heat diffusivity by the laser pulse [1] required strict 


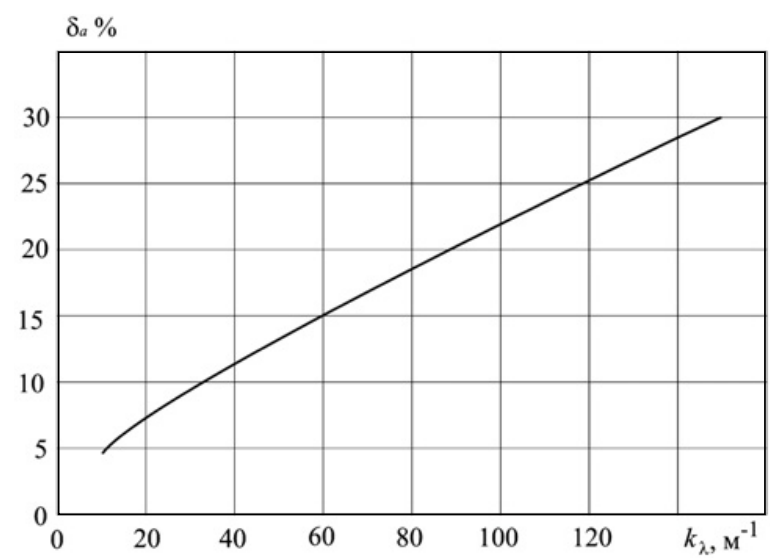

Figure 2. Dependence of the error $\delta_{a}$ of optical vitreous silica KU- heat diffusivity determination on absorption coefficient $k_{\lambda}$.

limits on the value of the wavelength of the incident radiation and the absorption coefficient. Error $\delta_{a}$ reduction up to minimum admissible values is possible at considerable reduction of laser radiation wave length in the field of translucency.

Therefore, the possibilities of substantial reduction of methodological errors of the laser pulse method in determining the heat diffusivity of vitreous silica are limited. At the same time it should be noted that the established by the results of theoretical studies variation error range of determination of heat transparent materials diffusivity (and hence other thermal characteristics) at moderate (up to $30 \mathrm{~m}^{-1}$ ) absorption coefficients are acceptable for many applications.

\section{Conclusion}

The values of systematic error, heat diffusivity, caused by radiative energy transfer as a result of mathematical simulation of heat transfer processes in silica glass under conditions corresponding to the implementation of a pulsed laser heating method of determining the thermal characteristics were calculated.

Expediency of values estimates of additional methodological errors which are due to material transparency, was justified at experimental design for determining the thermal characteristics of glasses for different purposes.

\section{References}

[1] Parker W. J. Flash method of determining heat diffusivity, heat capacity and thermal conductivity / W. J. Parker, R.J. Jenkins, C.P. Butler // J. Appl. Phys. -1961. - V. 32. - N. 9. - Pp. 1675-1684

[2] Akoshima, M. Study on a thermal-diffusivity standard for laser flash method measurements / M. Akoshima, T. Baba // Int. J. Thermophys. - 2006. - V. 27. - N. 4. - Pp. 1189 -1203

[3] Gaosheng W. Heat diffusivity measurements on insulation materials with the laser flash method / W. Gaosheng, Z. Xinxin, Y. Fan, C. Kui // Int. J. Thermophys. - 2006. - V. 27. - N. 1. Pp. 235- 243

[4] Heckman, E. M. Measurement of optical and thermal properties of $\mathrm{Hg}[1-\mathrm{x}] \mathrm{Cd}[\mathrm{x}] \mathrm{Te} / \mathrm{E} . \mathrm{M}$. Heckman, L. P. Gonzalez, S. Guha // Appl. Opt. - 2008. - V. 47. - N. 4. - Pp. 578-582 
[5] Novitsky L.A. Optical properties of materials at low temperatures / L.A. Novitsky, B.M. Stepanov. - M.: Mechanical Engineering, 1980. - P. 224

[6] Tovstonog V.A. Analysis of heat transfer in the light-scattering material is heated by radiation // Physics and chemistry of metal. - 1985. - No 3. - Pp. 35-40

[7] Prosuntsov P.V. Problems of heat transfer in a partially transparent scattering materials / P.V. Prosuntsov, S.V. Resnick // Sib. Physico-Technical. magazine. - 1991. - Vol. 1. - Pp. 51 - 56

[8] Prosuntsov P.V. Determination of thermophysical properties of translucent materials / P.V. Prosuntsov, S.V. Resnick // IFZh. - 1985. - V. 49. - Pp. 971 - 976

[9] Andre S. A theoretical study of transient coupled conduction and radiation heat transfer in glass: phonic diffusivity measurements by the flash technique / S. Andre, A. Degiovanni // Int. J. Heat Mass Transfer. - 1995. - V. 38. - N 18. - Pp. 3401-3412

[10] Kabayabaya T. Heat diffusivity Measurement of Glass at High Temperature by Using Flash Method / T. Kabayabaya, F. Yu, X. Zhang //J. of Thermal Science. - 2003. - V. 13. - N. 1. Pp. 91-96

[11] Merzlikin V.G. Method of processing the surface of translucent materials in pulsed mode / V.G. Merzlikin O.V. Sidorov, V.A. Tovstonog // Proceedings of the International Scientific Symposium "of tractor." - M. Bauman MAMI. - 2009. -Pp. 154-158

[12] Kuznetsov G. V. Pulse method systematic errors theoretical analysis for construction materials finite samples thermophysical characteristics determination / G. V. Kuznetsov, M. D. Kats // Measurement Techniques. - 2009. - V. 52. - N 4. Pp. 384-386

[13] Yavorsky B.M. Handbook of physics / B.M. Jaworski, A. Detlaf. - Moscow: Nauka, 1985. P. 512

[14] Samarskiy A.A. Theory of difference schemes. - Moscow: Nauka, 1983. - P. 616

[15] Kuznetsov G. V., Mamontov G.Ya. Taratushkina G. V. Numerical simulation of ignition of a condensed substance bu a particle heated to high temperatures. Combustion Explosion and shock Waves. 2004. V. 40. N. 1. P. 70-76

[16] Kuznetsov G. V., Strizhak P.A. 3D problem of heat and mass transfer at the ignition of a combustible liquid by a heater metal particle. Journal of Engineering thermophysics. 2009. V. 18. N. 1. P. $72-79$

[17] Kuznetsov G. V., Strizhak P.A. The influence of heat transfer condition at the hot -liquid fuel interface on the ignition characteristics. Journal of Engineering thermophysics. 2009. V. 18. N. 2. P. $162-167$ 